\title{
EVALUATING INTERACTIVE VISUALIZATION TECHNIQUES ON SMALL TOUCH SCREEN DEVICES
}

\author{
Muzammil Khan ${ }^{1}$, Sarwar Shah Khan ${ }^{2}$, Kifayat Ullah ${ }^{3}$, and Ghufran Ullah*4 \\ ${ }^{1,3}$ Department of Computer \& Software Technology, University of Swat, Pakistan \\ ${ }^{2}$ College of Information Science and Technology, Beijing University of Chemical \\ Technology, Beijing, China \\ ${ }^{4}$ Department of Computer Science, City University of Science \& IT, Pakistan \\ 1*muzammil@uswat.edu.pk, ${ }^{2}$ sskhan0092@gmail.com, ${ }^{3}$ kifayat@uswat.edu.pk, \\ and ${ }^{4 *}$ ghufran@ cusit.edu.pk
}

Abstract- The purpose of the study is to introduce interactive mechanisms for information visualization techniques, which will assist the nomadic users in exploring information on small touch screen mobile devices like smartphones and tablets, on the move. The study is carried out by choosing the Posttest-Only Randomized Experimental research design and evaluated by using a questionnairebased control experiment. The participants were asked to execute several tasks based on experiments on a functional prototype and fill the feature-based questionnaire. The study introduced six interactive visualization techniques to visualize information in a way that conveys the insight of the data effectively. The results indicate that Drill down approach in column chart (DDA+CC) and Legend navigation approach in column chart $(\mathrm{LNA}+\mathrm{CC})$ shows the reliable results and facilitate information manipulation on small screens of mobile devices, Drill down approach in bar chart (DDA+BC) and Legend navigation approach in bar chart (LNA+BC) shows weak results for Smartphones, the interactive visualization techniques DDA+LNA+CC and DDA+LNA+BC are highly appreciated for mobile devices, that are for both Smartphones and Tablets.

Keywords - Data mining result's interactivity, Drill down interactive visualization technique, Legend navigation interaction, Mobile device visualization interactivity

\section{INTRODUCTION}

The advancement in mobile technologies, telecommunication technologies, and the decreasing cost encourages the researchers to utilize battery-operated mobile devices for social interaction and information search using the World Wide Web (WWW). The researchers are prompt to work for efficient mobile interfaces that should effectively display information on small screen devices and efficiently utilize unreliable low bandwidth connections. Despite the revolutionary enhancement in the resources (computation, storage, connectivity, etc.) of mobile devices, the mobility and scaleddown technologies lead to some limitations [20].

Mobile devices can be used to facilitate the nomadic users to access information quickly, easily, and almost at no cost. Smartphones and tablets are widely used mobile devices, for information access and search, news, social interaction, eBooks, articles and

Received: June 8, 2019

Reviewed: December 9, 2019

Accepted: December 11, 2019

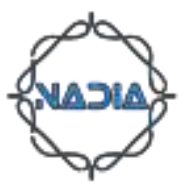


entertainment, etc., via Internet [10]. Mobile devices gained more popularity because of features like computation, memory, connectivity, etc., the researchers have been encouraging to adopt services from desktop systems or laptop computer systems to mobile devices.

On the other hand; data mining in the present era is attracting the attention of persons related to the information industry, marketing or business, investors, etc., even society as a whole. For example, to track the stocks in the stock market, to find market trends about a product, to find out the best book on the basis of some criterion in the Amazon book record repository to guide a user to take the decision and buy the book, etc.

Mobile data mining is the integration of data analysis techniques in the mobile computing environment. Mobile data mining can be marketed to facilitate a wide range of mobile device users with useful, potential innovative information extracted with advanced data mining techniques. However, comprising these emerging technologies is not that simple and straightforward, hence facing a number of challenges.

\subsection{VISUALIZING MINING RESULTS ON MOBILE DEVICES}

For ubiquitous use of data mining techniques and rapid development in mobile technology, encourages researchers to introduce techniques that utilize these emerging technologies [4]. Datasets for data mining are normally very huge, need high storage capacities, the data mining processes or tasks are computationally very expensive and need high computation capabilities. Thus it is not feasible to bring data mining tasks to mobile devices having limited resources. A study comprehensively discussed possible scenarios, where we can incorporate data mining techniques in mobile devices and the best feasible architecture in the environment with current resources of mobile devices [14].

The best way is to provide beneficial data mining results (required extracted information) in such a way that they are more useful to the end-user and according to their cognitive capabilities. The client-server architectures [14] allow mobile devices to invoke, execute data mining tasks on the remote server from the client mobile device and visualize or demonstrate significant mining results to the nomadic users. Keeping in view the importance of data mining, the use and limitations of mobile devices, the demand is to effectively visualize results (information) on a small screen of a mobile device. The information should be visualized in such a way that it provides the inside in a glance, easy to understand with less cognitive resources and less distraction of the user from their current task [5]. The human cognitive capabilities enable the user to work on several tasks at a time or in parallel and demand fast information retrieval and processing, effective and easy to complete.

The research statement of the study is "Interactive and usable visualization techniques for data mining results on mobile devices can easily and effectively interpret the inside of data".

\subsection{HOW TO VISUALIZE ON SMALL SCREEN DEVICES?}

The information display on the small screen of the mobile device faces various challenges due to the limited size screen and the absence of standard input devices that make it more difficult to introduce effective visualization techniques. To produce productive visualization techniques [14], we need to focus and deal with the information as follow;

- Information selection: As mobile devices have small screens, so it is important to use them wisely and select information, which is of utmost importance for the users.

- Information Presentation: To display information on small screen offers more challenges with mobile devices and become the most important problem to handle, 
and select information presentation or visualization technique that best suited the nature of the data to be presented.

- Interface Interactivity: To manage information on the available small screen, need efficient and effective interactive mechanisms to manipulate information for the users effectively.

- Visualization Features: To produce effective information visualization techniques and to ensure the visualization features like functionality, usability, effectiveness, efficiency, and usefulness.

\section{FEATURES AND OBJECTIVES}

The objectives of this study are to achieve the intended features (discussed subsequently) by developing a functional prototype in order to validate the proposed framework, which facilitates mobile device users with interactive, useful and informative information [14]. The features are briefly discussed below;

\subsection{DYNAMIC AND SCALABLE LAYOUT}

The layouts of the functional prototype dynamically transform the visual layout or information visualization regarded mobile device screen size. The scalability of visualization techniques make it more feasible for small size screen, the zooming (zoom in or zoom out) should not distort the graphics and provide a sharp and clear visual graphics display on mobile devices. Scalability and dynamism both are technological issues; these features can be achieved with the help of advanced technologies, by considering best-suited programming languages and techniques after analyzing the implementation environment. As shown in Figure 1.

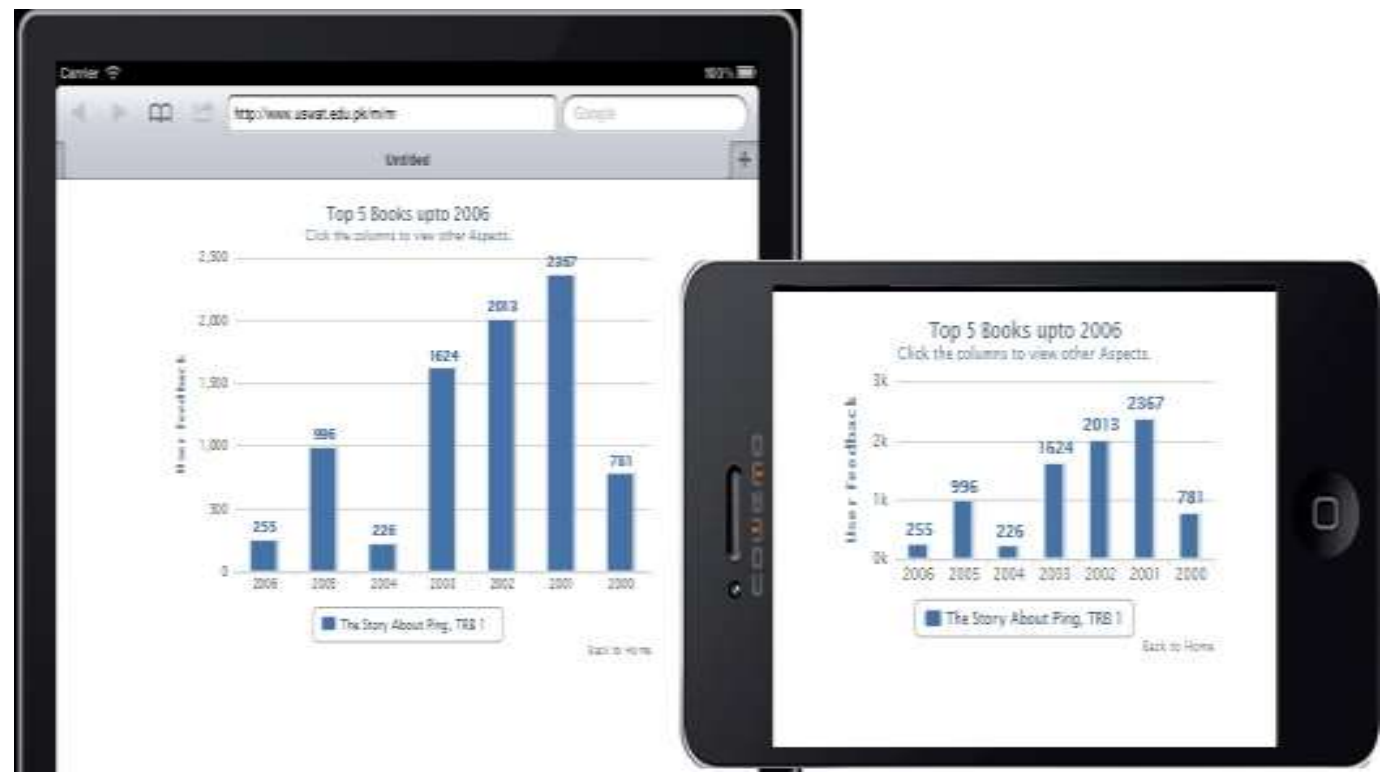

Fig. 1 DDA Interaction Visual Display on Smartphone and Tablet

\subsection{VISUALIZATION TECHNIQUES}

In literature, a number of visualization techniques available [12]. The selection of visualization technique depends on the nature of the data to be visualized [18], this study considered two visualization techniques, like column chart and bar charts because these are best by representing information initiated by data discrimination, data characterization 
[7] and the potential information that can be extracted from the selected Amazon dataset [8].

\subsection{INTERACTIVE MECHANISMS}

The Amazon dataset is explored for the best book based on different criteria by extracting related information, the focus is on the following three interactive mechanisms.

- Drill Down Approach: Accessing low-level details of the data from a higher level or drill down means to move from abstract or aggregated information to detailed information, which is extracted via data mining tasks in the mobile data mining environment [11].

- Legend Navigation Approach: The legend navigation approach is the best-suited mechanism for data discrimination, i.e. to compare the comparative target class(es) [13].

- Drill down + Legend Navigation Approach: To get comprehensive details about target classes and explore intended information by both dimensions, i.e. exploring one information graphical representation from a higher level to the low level and comparing it with other comparative classes.

\subsection{VISUALIZATION FEATURES}

Effective information visualization techniques should support visualization features like functionality, usability, effectiveness, efficiency, and usefulness. Visualization features have been validated by evaluating the functional prototype using empirical evaluation and posttest-only randomized experimental research design.

\subsection{MULTIPLE BRAND SMARTPHONES AND TABLETS SUPPORT}

The functional prototype supports different brands of smartphones and tablets regardless of their underlying architecture. The web browser is used for interactive visualization techniques presentation as data mining results become available to a wide range of mobile device users. For evaluation different brands of smartphones and tablets are employed during experiments to rectify technical issues in the information presented.

\section{RELATED WORK}

The main purpose of mobile data mining is to provide data mining techniques for the analysis and monitoring of data from mobile devices [24]. As mobile devices are now more common in use and the mobile technologies prevail around. The focus is on the mobile device involvement with data mining techniques either directly or indirectly. Direct involvement means to act as a mining machine and perform mining tasks on a mobile device. Indirect involvement means that the mobile device act as a data provider or consumer, whether the mobile device is used to provide data to the mining machine or used to invoke data mining tasks and provide data mining produced information to the end-user.

There are several studies that exist regarding mobile data mining. [9] introduced a mobile data mining system MobiMine for monitoring current trends in stock market, [17] enhanced MobiMine and specified the detection of dependencies among different features, [27] proposed data mining systems for distributed and huge data, [3] developed a VEhicle DAta Stream mining (VEDAS) system, for monitoring and mining real-time vehicle data streams, PDM [21] is a data mining system describing collaborative mining of streaming data in mobile and distributed computing environments, [22] introduced new version of Pocket Data Mining (PDM) system for data stream classification in the distributed pocket data mining system, [23] proposed the use of two classifiers, Hoeffding trees and Naive 
Bayes for distributed data stream mining. Mobile devices can be used for traditional data mining, stream mining or for real-time behavior detection, etc. For example, a new scoring mechanism that receives data streams from the driver's smartphone through vehicle sensors to performed intrinsic evaluation to assess and detect driver's driving behaviors using three outlier detecting algorithms [26]. Table 1 shows the comparison of different features related to the study being performed for mobile data mining. The top row in Table I represents the mobile data mining systems and the leftmost column represents different features in these systems. "Year" means the year of publishing, "Supported Mobile devices", "Different Mobile devices Support", means that the type of devices supported the system. "Domain" from where the data set has been considering for the experiments. "Server-Side Mining" or "Client-Side Mining" where the mining machine has been implemented, and client means the mobile device, "Designated community" means providing access to limited or general peoples.

Table I. Feature Summarization of Mobile Data Mining Systems

\begin{tabular}{|c|c|c|c|c|c|c|c|c|}
\hline $\begin{array}{l}\text { Mobile Data } \\
\text { Mining } \\
\text { System }\end{array}$ & $\begin{array}{l}\text { MobiMine } \\
\text { (Monitoring } \\
\text { stock } \\
\text { market) }\end{array}$ & $\begin{array}{c}\text { Enhanced } \\
\text { MobiMine } \\
\text { (Dependency } \\
\text { detection) }\end{array}$ & $\begin{array}{l}\text { VEDAS } \\
\text { (Vehicle } \\
\text { data } \\
\text { stream } \\
\text { mining) }\end{array}$ & $\begin{array}{c}\text { PDM } \\
\text { (Collaborative } \\
\text { mining) }\end{array}$ & $\begin{array}{c}\text { PDM } \\
\text { (Datastream } \\
\text { classification) }\end{array}$ & $\begin{array}{c}\text { PDM } \\
\text { (Two } \\
\text { classifiers) }\end{array}$ & $\begin{array}{c}\text { DDA } \\
\text { (Best } \\
\text { Book } \\
\text { Selection) }\end{array}$ & $\begin{array}{c}\text { LNA } \\
\text { (Best } \\
\text { Book } \\
\text { Selection) }\end{array}$ \\
\hline Year & 2002 & 2003 & 2005 & 2010 & 2010 & 2011 & 2014 & 2016 \\
\hline $\begin{array}{l}\text { Supported } \\
\text { Mobile } \\
\text { Devices }\end{array}$ & PDA & PDA & PDA & $\begin{array}{c}\text { Mobile } \\
\text { Software } \\
\text { Agent }\end{array}$ & $\begin{array}{c}\text { Mobile } \\
\text { Software } \\
\text { Agent }\end{array}$ & $\begin{array}{c}\text { Mobile } \\
\text { Software } \\
\text { Agent }\end{array}$ & ALL & $\overline{A L L}$ \\
\hline $\begin{array}{l}\text { Different } \\
\text { Mobile } \\
\text { Device } \\
\text { Support } \\
\end{array}$ & $\mathrm{NO}$ & $\mathrm{NO}$ & $\mathrm{NO}$ & $\mathrm{NO}$ & Maybe & Maybe & YES & YES \\
\hline Domain & $\begin{array}{l}\text { Stock } \\
\text { Market }\end{array}$ & $\begin{array}{c}\text { Distributed } \\
\text { Data Mining }\end{array}$ & $\begin{array}{l}\text { Vehicle } \\
\text { Fleet } \\
\text { Mining }\end{array}$ & NA & $\begin{array}{c}\text { UCI } \\
\text { Repository } \\
\text { Data Sets }\end{array}$ & $\begin{array}{c}\text { UCI } \\
\text { Repository } \\
\text { Data Sets }\end{array}$ & $\begin{array}{c}\text { Amazon } \\
\text { Book } \\
\text { Dataset }\end{array}$ & $\begin{array}{c}\text { Amazon } \\
\text { Book } \\
\text { Dataset }\end{array}$ \\
\hline $\begin{array}{l}\text { Mining } \\
\text { Method }\end{array}$ & $\begin{array}{c}\text { Data } \\
\text { Stream } \\
\text { Mining }\end{array}$ & $\begin{array}{c}\text { Association } \\
\text { Rule Mining }\end{array}$ & $\begin{array}{c}\text { Data } \\
\text { Stream } \\
\text { Mining }\end{array}$ & $\begin{array}{l}\text { Collaborative } \\
\text { Data Mining }\end{array}$ & Data Mining & Data Mining & $\begin{array}{c}\text { Descriptive } \\
\text { Data } \\
\text { Mining }\end{array}$ & $\begin{array}{c}\text { Descriptive } \\
\text { Data } \\
\text { Mining }\end{array}$ \\
\hline $\begin{array}{l}\text { System } \\
\text { Architecture }\end{array}$ & $\begin{array}{l}\text { Client- } \\
\text { Server }\end{array}$ & $\begin{array}{l}\text { Client- } \\
\text { Server }\end{array}$ & $\begin{array}{l}\text { Client- } \\
\text { Server }\end{array}$ & Stand alone & Client-Server & Client-Server & $\begin{array}{l}\text { Client- } \\
\text { Server }\end{array}$ & $\begin{array}{l}\text { Client- } \\
\text { Server }\end{array}$ \\
\hline $\begin{array}{l}\text { Server-side } \\
\text { Mining }\end{array}$ & YES & YES & $\mathrm{NO}$ & NA & YES & YES & YES & YES \\
\hline $\begin{array}{l}\text { Client side } \\
\text { Mining } \\
\end{array}$ & $\mathrm{NO}$ & $\mathrm{NO}$ & YES & YES & $\mathrm{NO}$ & $\mathrm{NO}$ & $\mathrm{NO}$ & $\mathrm{NO}$ \\
\hline $\begin{array}{l}\text { Mining } \\
\text { Algorithms }\end{array}$ & $\begin{array}{c}\text { Correlation } \\
\& \\
\text { Conditional } \\
\text { Probability } \\
\text { Rule } \\
\end{array}$ & $\begin{array}{l}\text { Distributed } \\
\text { Scanone }\end{array}$ & K-means & KNN & Naive Bayes & $\begin{array}{c}\text { Hoeffding } \\
\text { Tree }\end{array}$ & Correlation & Correlation \\
\hline $\begin{array}{l}\text { Results } \\
\text { Representation }\end{array}$ & $\begin{array}{l}\text { Tabular \& } \\
\text { Treemap }\end{array}$ & NA & $\begin{array}{c}\text { Polygonal } \\
\& \\
\text { Line } \\
\text { graph }\end{array}$ & NA & $\begin{array}{c}\text { Classification } \\
\text { Tree }\end{array}$ & $\begin{array}{c}\text { Classification } \\
\text { Tree }\end{array}$ & $\begin{array}{c}\text { Bar and } \\
\text { Column } \\
\text { Charts }\end{array}$ & $\begin{array}{l}\text { Bar and } \\
\text { Column } \\
\text { Charts }\end{array}$ \\
\hline
\end{tabular}

\section{EXPERIMENTAL SETUP}

\subsection{APPLICATION SCENARIO}

The studies [14] and [10] discusses six different application scenarios, where we can assimilate data mining in mobile devices environment based on the role of a mobile device. As discussed the limitations of the mobile device, the most prominent role is to use it as a mobile client or data provider. For example, the [19] discusses the role of the mobile device as a data collector from citizen's mobile communication and use for 
intelligent transportation management for city planners. Similarly, the VisTiles framework presents a mobile device as a tool to explore visual data by coordinating and combining co-located mobile devices [15]. Keeping in mind the mobile device restraints, massive datasets, and high computing data mining techniques or processes, $1 \mathrm{st}$ and $3 \mathrm{rd}$ scenarios are best to employ. In both the scenarios, the mobile device acts as a client to view mining results and facilitate the nomadic user providing interactive visualization techniques, as shown in Figure 2 and Table II.

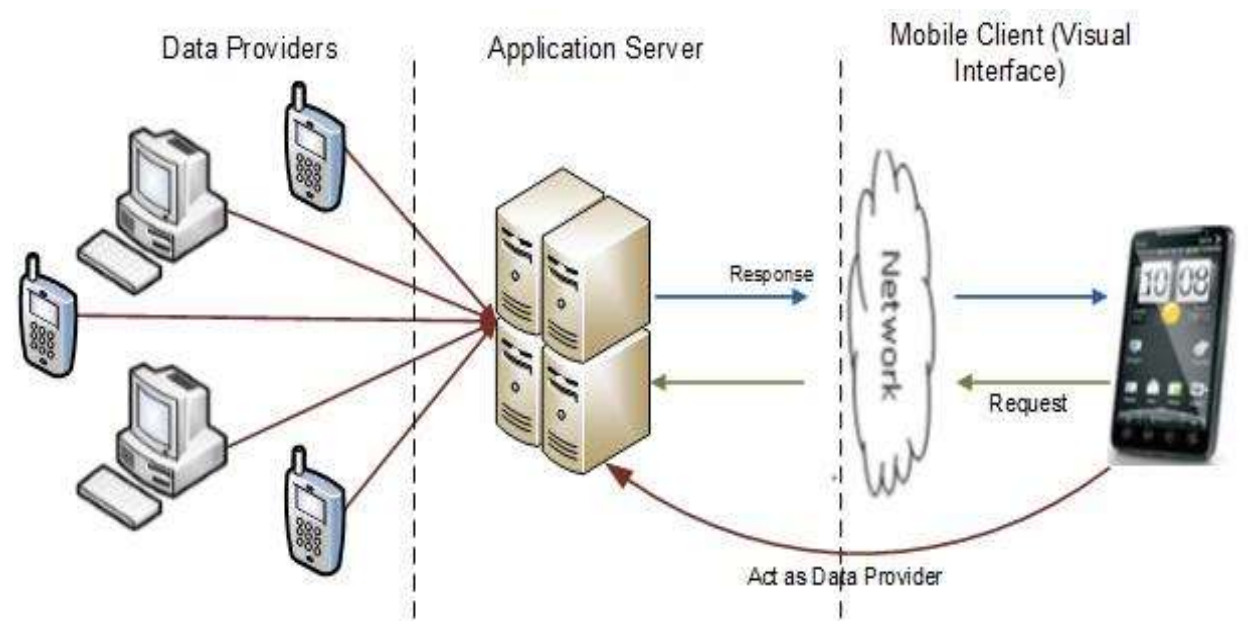

Fig. 2 Mobile Data Mining Application Scenario

Table II. 3rd Scenario: Mobile Devices as Data

\begin{tabular}{|l|ll|l|}
\hline Data Providers & \multicolumn{2}{|l|}{ Application Server } & Mobile Client \\
\hline$\bullet \quad$ Provide data & $\bullet \quad$ Perform Mining Tasks & $\bullet$ & Visual Interface \\
& $\bullet \quad$ Respond Mobile Client Requests & $\bullet$ & Request Mining Results \\
& $\bullet \quad$ Data Storage (Dataset) & $\bullet$ & Data Provider \\
\hline
\end{tabular}

\subsection{RESEARCH DESIGN}

Research design is one of the essential parts of any research study and used to carefully and efficiently investigate the final outcome. Without proper research design, the study may conclude weak results and even fail to answer the questions raised in the framework. "Research design in the plan, structure, and strategy of investigation conceived so as to obtain answers to research questions and to control variance" [10].

A number of research designs are discussed in different studies [10][14]. For this study, we choose the Posttest-Only Randomized Experimental research design because we do not have any definite interactive visualization techniques or studies for mobile devices to which can be used for comparisons.

\subsection{EVALUATION TECHNIQUES}

The worth, reliability and significance of a research study can be analyzed by choosing a suitable evaluation procedure based on some criteria. Evaluation is one of the substantial steps of the research process [16]. The selection of evaluation technique depends on the nature of the research study. A number of techniques are discussed in [8]. The study evaluates the effectiveness of the interactive technique in information visualization by considering visualization features, briefly described as;

- Functionality: Up to what extent the visualized information presents the functionalities compelled by the end-users? 
- Effectiveness: How the information visualization techniques are advisable (valuable, meaningful) and convey the insight effectively?

- Efficiency: Up to what extent the visualization techniques provide help to the users and lead to achieve the intended or expected results with better performance?

- Usability: The visualization techniques provide easy user interaction and should be clear and understandable.

- Usefulness: Are the visualization techniques is beneficial, what is the overall user perception?

This study is evaluated by using a questionnaire-based control experiment. In questionnaire-based control experiments, the participants were asked to execute a number of task-based experiments using functional prototype and fill the feature-based questionnaire and conduct individual interviews.

\subsection{PARTICIPANTS}

Target Population: The individuals who have experienced the Amazon online retailer, observed the best book and have used or are using a mobile device(s) like Smartphone \& Tablet, make up our target population.

Accessible Population: The students from different educational institutions around the capital and Khyber Pakhtunkhwa are considered, which have experience the Amazon online retailer, the best book and have used or are using a mobile device(s), make up our accessible population.

Sampling: The study considered the simple random sampling technique, where each individual from the population has an equal chance of being selected as a sample.

To evaluate the posted research statement with real users by applying the functional prototype, the study included 41 volunteer participants. The participant's demographics are summarized on the bases of age and education, as follows;

Table III. Participant's Demographics on the bases of Age and Education

\begin{tabular}{|l|l|l|}
\hline \multicolumn{3}{c}{ Age } \\
\hline Age Range & Male (73\%) & Female (27\%) \\
\hline $21-25$ & $19 \%$ & $14 \%$ \\
\hline $26-30$ & $41 \%$ & $10 \%$ \\
\hline $31-35$ & $13 \%$ & $03 \%$ \\
\hline \multicolumn{3}{|l|}{ Education } \\
\hline Education Degree & Male (73\%) & Female (27\%) \\
\hline BS & $12 \%$ & $05 \%$ \\
\hline MS & $41 \%$ & $15 \%$ \\
\hline PhD & $20 \%$ & $07 \%$ \\
\hline
\end{tabular}

The participants were selected for experiments based on two demographic parameters, first; age and second; education, using the mobile device. The education of the selected participant is more important than all other demographic characteristics because the selected dataset is an Amazon book dataset where the selection is to be done by an individual from an educated background, observed Amazon books shopping and having experience of mobile devices. 


\subsection{DATASET}

In the study, the data mining techniques are applied to the Amazon Books dataset published in 2008 [8]. The dataset has 7 years of data from the year 2000 to the year 2006, the size of the dataset is $8.3 \mathrm{~GB}$, contains user's feedback or reviews, helpful feedback, book ratings, user's ranking, etc. The tasks have been designed to find out the best book on the bases of some specified criterion.

\subsection{SURVEY DESIGN}

To evaluate the study we choose the control experiment evaluation technique based on survey or questionnaire. Quantitative research methods are useful to identified relationships among variables, measure and analyze using statistical techniques [10]. After studying numerous systems [25] conclude that $78 \%$ of the evaluation is based on surveys. In surveys, a questionnaire is used to collect the data using a functional prototype over a sample of real users selected from the population and generalize the results to the whole population [1]. The questionnaire used for the evaluation contained five features, visualization techniques, and interactive mechanisms, using a five-point Likert scale which is commonly used in questionnaires and is the most widely used scale in survey research [6].

Five-point Likert Scale

\section{Strongly Agree 4. Agree 3. Neutral 2. Disagree 1. Strongly Disagree}

The questionnaire contains questions related to different tasks. The main goal of the study is to provide interactive usable visualization techniques for data mining results to ensure the best way to convey information inside the huge data by minimal efforts and help to identify the best book on the bases of different criteria using tasks. The selection of these tasks based on the potential information available and extracted from the dataset. Tasks are about to "Find out the best book on the bases of some criteria", as explained in Table IV.

Table IV. Tasks Details and Interactive Mechanisms Applied

\begin{tabular}{|c|l|l|}
\hline $\begin{array}{l}\text { Task } \\
\text { No }\end{array}$ & Tasks & Interactive Visualization Technique \\
\hline 1 & $\begin{array}{l}\text { Best book on the bases of getting the maximum number } \\
\text { of feedbacks or user response }\end{array}$ & $\begin{array}{l}\text { Drill Down Approach and Column } \\
\text { Chart }\end{array}$ \\
\hline 2 & $\begin{array}{l}\text { Best book on the bases of getting the maximum number } \\
\text { of positive feedbacks }\end{array}$ & Drill Down Approach and Bar Chart \\
\hline 3 & $\begin{array}{l}\text { Best book on the bases of High percent of good } \\
\text { feedback with the highest feedback }\end{array}$ & $\begin{array}{l}\text { Legend Navigation Approach in } \\
\text { Column Chart }\end{array}$ \\
\hline 4 & $\begin{array}{l}\text { Best book on the bases of High percent good feedback } \\
\text { with the highest rating }\end{array}$ & $\begin{array}{l}\text { Legend Navigation Approach in Bar } \\
\text { Chart }\end{array}$ \\
\hline 5 & $\begin{array}{l}\text { Best book on the bases of 100\% good feedback with the } \\
\text { highest feedback }\end{array}$ & $\begin{array}{l}\text { Drill Down +Legend Navigation } \\
\text { Approach in Column Chart }\end{array}$ \\
\hline 6 & $\begin{array}{l}\text { Best book on the bases of 100\% good feedback with the } \\
\text { highest feedback with a high rating }\end{array}$ & $\begin{array}{l}\text { Drill Down +Legend Navigation } \\
\text { Approach in Bar Chart }\end{array}$ \\
\hline
\end{tabular}

\section{RESULTS ANALYSIS AND DISCUSSION}

Evaluation is an essential part of the research process and is a systematic approach to analyze the worth, quality, reliability and assess the significance of the research study by analyzing the data. In user-centric based studies evaluation is to validate the posted research statement by using statistical measures. 


\subsection{ANALYSIS PROCEDURE}

The evaluation of visualization techniques on mobile devices provided via a functional prototype is presented to participants. To analyze visualization techniques with different related features on mobile devices, the survey is conducted through a questionnaire from the participants. The participants were asked to perform tasks on the Smartphone and tablet. On the completion of tasks, to accomplish the conducting survey and fill the given questionnaire, the main points according to [10] are;

- A group, sample, representing the population is studied for their characteristics (such as abilities, opinions, attitudes, beliefs, and/or knowledge) to extract information.

- Data is collected from the individuals by mainly asking questions using feedback forms, i.e. questionnaire.

- Only the sample is asked a question rather than going through all the members of the population.

The user's responses for each combination have noted based on directly and indirectly effective parameters or features of visualization techniques. Its related statistical measures are computed by three means;

- Mean is computed to find out the average user's responses for each question about a feature for the overall user's perception.

- Median is computed to show the most selected option from the Likert scale and hence justifies the mean value.

- Standard Deviation is required to show the variation among users responses, high standard deviation means high variation in user's responses and hence less reliable results or vice versa.

\subsection{VISUALIZATION FEATURES}

This part of the article assesses the extent to which the visualization techniques promising the quality of the visualization features. In Figure 3, the interactive mechanisms are compared for each feature and briefly discussed below;

5.2.1. FUNCTIONALITY: The participants were asked five questions to measure this feature and some complementary questions in the interview to understand it better.

Table V. Mean, Median, Standard Deviation measures for Functionality

\begin{tabular}{|l|l|l|l|l|l|}
\hline Measure & Q1 & Q2 & Q3 & Q4 & Q5 \\
\hline Mean & 4.52 & 4.58 & 4.55 & 4.65 & 4.12 \\
\hline Median & 5 & 5 & 5 & 5 & 4 \\
\hline Standard Deviation & 0.63 & 0.56 & 0.57 & 0.54 & 0.59 \\
\hline
\end{tabular}

In question Q1, the participants were asked about the understandability and easiness of the functionality provided in visualization techniques, 58\% of the participants strongly agreed and 35\% agreed. In question Q2, the participants are asked about layout functionality, e.g. appearance, information dissipation, etc., $61 \%$ strongly agreed and $35 \%$ are agreed. In question Q3, 58\%, 38\% of the participants are strongly agreed and agreed respectively that the visualization provides enough information. $70 \%$ of the participants strongly agreed that the zooming function does not distort the quality of the proposed technique, i.e. question Q5 feedback. In Q6, the participants were asked about axes labels, data labels enhance understanding capacities, $65 \%$ of the participants agreed. 
Comparisons of these techniques are of great importance, to evaluate how much the interactive visualization techniques present the functionality according to the user's requirement. Figure 3 shows the functionality of the proposed techniques. The users have a similar response towards all the techniques and show constant results. The mean value varies between $\mathrm{M}=3.84$ (Median=4, $\mathrm{SD}=0.52$ ) for $\mathrm{LNA}+\mathrm{BC}$ and $\mathrm{M}=4.38$ (Median=4, $\mathrm{SD}=0.55$ ) for $\mathrm{DDA}+\mathrm{LNA}+\mathrm{CC}$, which is not a significant difference, shows that the techniques presented, serve the users best to complete the tasks.

5.2.2. EFFECTIVENESS: To measure this feature the participants were asked five questions and a few complimentary questions.

Table VI. Mean, Median, Standard Deviation measures for Effectiveness

\begin{tabular}{|l|l|l|l|l|l|}
\hline Measure & Q7 & Q8 & Q9 & Q10 & Q12 \\
\hline Mean & 4.61 & 4.48 & 4.32 & 4.48 & 4.54 \\
\hline Median & 5 & 5 & 4 & 4 & 5 \\
\hline Standard Deviation & 0.48 & 0.61 & 0.58 & 0.5 & 0.49 \\
\hline
\end{tabular}

In Q7, the users were asked about the overall attractiveness of visualization techniques, $61 \%$ of the users strongly agreed with the fact. Questions Q9 and Q11 are about to keep the users focus and not distract from the primary goal while manipulating the visualization techniques, more than $75 \%$ are strongly agreed.

Effectiveness is one of the important measures of visualization that need to provide insight valuable information effectively. Figure 3 shows the effectiveness of the proposed techniques. The figure shows variable results for the techniques, the attractiveness of the technique $\mathrm{LNA}+\mathrm{BC}(\mathrm{M}=2.74$, Median=3, $\mathrm{SD}=0.56)$ and $\mathrm{DDA}+\mathrm{BC}(\mathrm{M}=3.06$, Median=3, $\mathrm{SD}=0.55)$ presented reasonably weak results. The usability factors are not prominent, while the DDA+LNA in bar charts $(\mathrm{M}=3.97$, Median $=4, \mathrm{SD}=0.68)$ and in column charts $(\mathrm{M}=4.35$, Median $=4, \mathrm{SD}=0.53)$ are appraised.

After experiments, It has been observed by discussing the effectiveness of the proposed techniques with the participants and concluded that the use of bar charts is not common and is the main reason to show the weak results as shown in the figure. For example, $\mathrm{LNA}+\mathrm{BC}, \mathrm{DDA}+\mathrm{BC}$, and the $\mathrm{LNA}+\mathrm{CC}$ presented borderline performance (specified by the red border in the figure).

5.2.3. EFFICIENCY: To measure this feature the participants were asked three questions and some complementary questions.

Table VII. Mean, Median, Standard Deviation measures for Efficiency

\begin{tabular}{|l|l|l|l|}
\hline Measure & Q14 & Q15 & Q16 \\
\hline Mean & 4.45 & 4.58 & 4.51 \\
\hline Median & 5 & 5 & 5 \\
\hline Standard Deviation & 0.62 & 0.62 & 0.5 \\
\hline
\end{tabular}

In question Q14, the participants were asked about learning and understandability of the manipulation of techniques, $90 \%$ of the participants agreed that the visualization techniques are not taking longer to learn and understand. They are very optimistic about data loading and inner information exploration, i.e. questions Q15 \& Q16.

The visualization techniques demand an effective, usable interface to interact with but not at the cost of efficiency. The efficiency is to achieve the goal with less resource usage, i.e. cost, effort and most importantly time. Figure 3 shows the average user's response of the participants for the interactive techniques defined in the proposed framework. The 
figure shows less variation and the most stable results for the proposed techniques. The user's response shows that these techniques are not taking a long time to learn, fetching inner information and learn how to manipulate it. The mean varies between $M=3.9$ to $\mathrm{M}=4.21$, Median=4 and very sustainable standard deviation, i.e. on average $\mathrm{SD}=0.53$.

5.2.4. USABILITY: To measure usability the participants were six questions and some complementary questions.

Table VIII. Mean, Median, Standard Deviation measures for Usability

\begin{tabular}{|l|l|l|l|l|l|l|}
\hline Measure & Q18 & Q19 & Q20 & Q21 & Q22 & Q23 \\
\hline Mean & 4.58 & 4.67 & 4.29 & 4.66 & 4.51 & 4.16 \\
\hline Median & 5 & 5 & 4 & 5 & 5 & 4 \\
\hline Standard Deviation & 0.56 & 0.47 & 0.52 & 0.47 & 0.51 & 0.58 \\
\hline
\end{tabular}

The majority of the participants are strongly agreed that the interface is easy to use, easy to learn, easy to explore different sections of the visualization and more than $80 \%$ of the participants agreed, related questions are Q 18, Q 19, Q 20. In questions, Q 21 and Q 22 , the user's response is encouraging about other usability aspects like data label, axes readability, color visibility, etc. Usability measure is vital for any user interface evaluation. The features like Learnability, understandability, memorability, ease of use and user satisfaction are the main features that are considered in usability studies. Figure 3 shows how much the techniques are usable for the end users after evaluation.

In Figure 3, the usability quality components are not prominent for bar charts and the interactive techniques in combination with bar charts presented weak results. For example, the technique $\mathrm{LNA}+\mathrm{BC}(\mathrm{M}=2.45, \mathrm{Median}=2, \mathrm{SD}=0.56)$ and $\mathrm{DDA}+\mathrm{BC}(\mathrm{M}=2.83$, Median=3, $\mathrm{SD}=0.52$ ) reveal feeble outcome, on contrast the DDA+LNA in bar charts $(\mathrm{M}=3.96$, Median=4, $\mathrm{SD}=0.71)$ and $\mathrm{DDA}+\mathrm{LNA}$ in column charts $(\mathrm{M}=4.36$, Median=4, $\mathrm{SD}=0.55)$ exhibit respectable usability quality.

\subsubsection{USEFULNESS}

Usefulness is the assurance of the relevance and quality of the information. It measures whether the application is useful in accomplishing the end-users tasks or not. Questions Q25, Q27 and Q28 are related to the information relevance and utility. 90\% of the participants are agreed to the information relevancy and utility. To measure this feature the participants were asked some complementary questions.

Table IX. Mean, Median, Standard Deviation measures for Usefulness

\begin{tabular}{|l|l|l|l|}
\hline Measure & Q25 & Q27 & Q28 \\
\hline Mean & 4.81 & 4.61 & 4.67 \\
\hline Median & 5 & 5 & 5 \\
\hline Standard Deviation & 0.40 & 0.55 & 0.54 \\
\hline
\end{tabular}

The usefulness measure evaluates the overall benefits from the user's point of view and the quality of information. Figure 3 describes how much visualization techniques are advantageous for end-users. The figure shows that the visualization techniques offer the same level of benefits and assist the users to achieve the intended goal. The mean value ranges between $\mathrm{M}=4.06$ (Median=4, $\mathrm{SD}=0.68$ ) for $\mathrm{LNA}+\mathrm{BC}$ and $\mathrm{M}=4.51$ (Median=5, $\mathrm{SD}=0.5$ ) for $\mathrm{DDA}+\mathrm{LNA}+\mathrm{CC}$, which is not a significant difference, shows that the techniques are best to complete the tasks. 


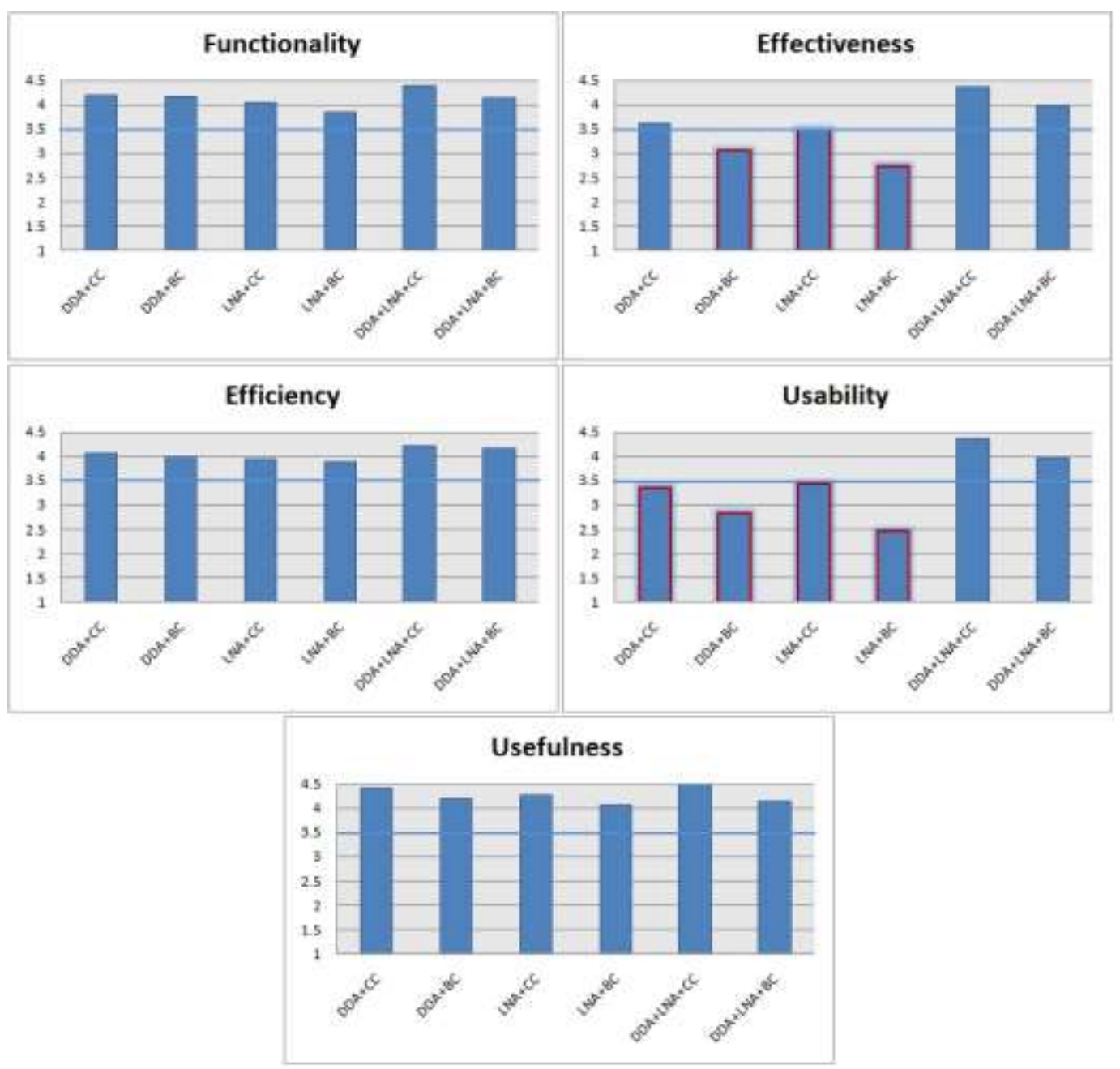

Fig. 3 Features Comparison of Interactive Visualization Techniques

\subsection{INTERACTIVE MECHANISMS \& VISUALIZATION TECHNIQUES}

The preceding section specifies the user's response to six different combinations of interactive mechanisms. Each combination has related features of visualization techniques and related measures mean median and standard deviation. The visualization features in interactive mechanisms are summarized in Figure 4. The blue line at value 3.5 indicates the user satisfaction level.

5.4.1. DRILL DOWN APPROACH IN COLUMN CHART: There are five different questions addressing five visualization features. The usage of the drill-down approach in the column chart shows stable results for all features. Approximately $85 \%$ of the participants agree with the functionality of this approach (Q 4.1), 65\% of the participants agree for effectiveness (Q 8.1), 85\% for efficiency (Q 13.1), 49\% for usability (Q 24.1), and 90\% shows their agreement for usefulness (Q 26.1). 


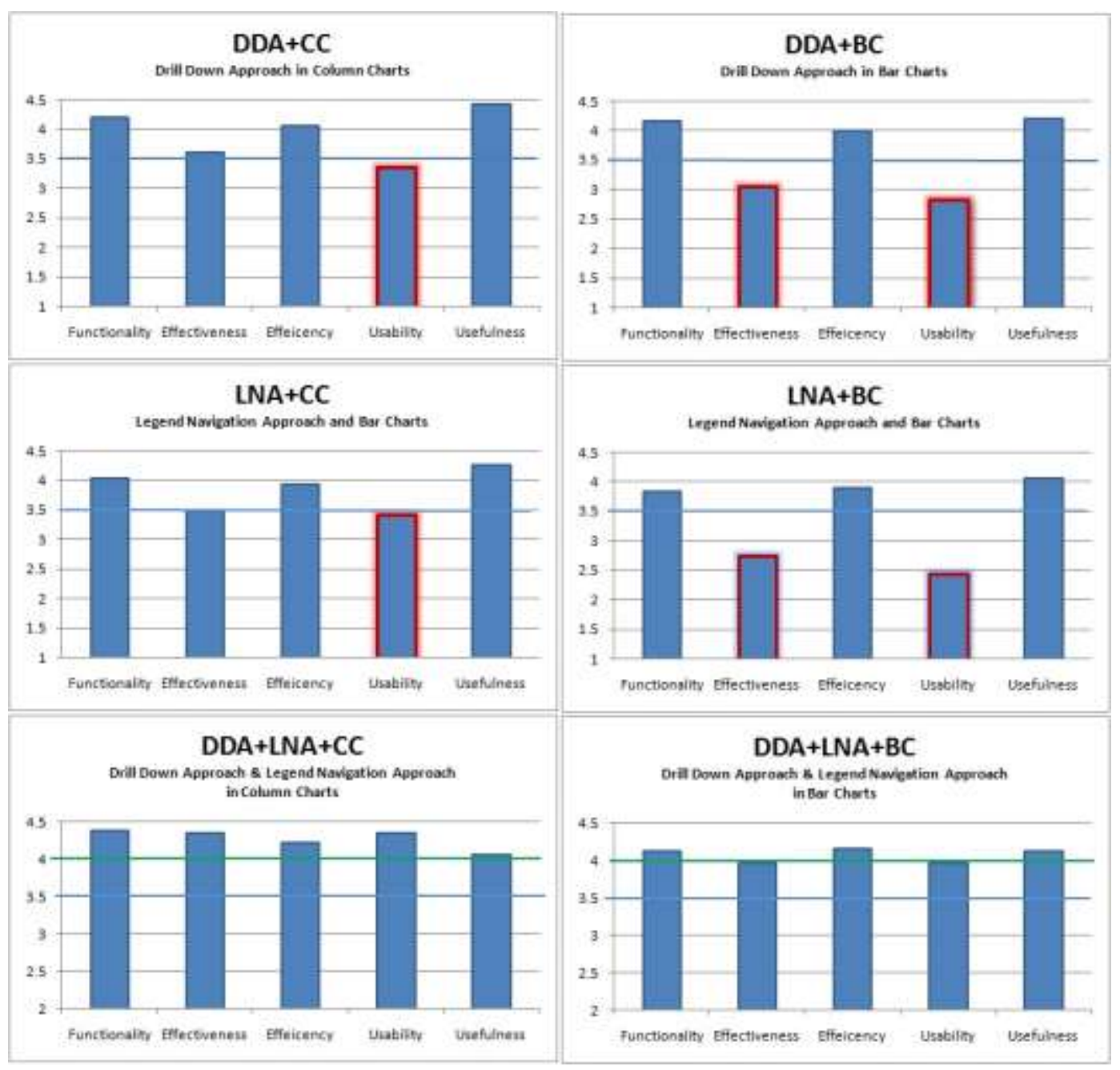

Fig. 4. Visualization Features in Interactive Mechanisms

Figure 4 shows the effectiveness of visualization features for a drill-down approach in column charts. The length of the bars corresponds to the mean values of the user's responses. Standard deviation ranges ( 0.55 to 0.65$)$, which shows the consistency of the user's responses. All the features present fair results.

5.4.2. DRILL DOWN APPROACH IN BAR CHART: The drill-down approach in the bar chart shows weak results for effectiveness and usability while other features show good results. Almost $80 \%$ of the participants agree for the functionality feature (Q 4.2), $49 \%$ for effectiveness (Q 8.2), 83\% for efficiency (Q 13.2), 41\% for usability (Q 24.2), and $79 \%$ for usefulness (Q 26.2).

Figure 4 shows the effectiveness of visualization features for a drill-down approach in bar charts. The length of the bars corresponds to the mean values of the user's responses. Standard deviation ranges ( 0.51 to 0.74$)$, which shows the consistency of the user's responses. The functionality, efficiency and usefulness features show promising results.

5.4.3. LEGEND NAVIGATION APPROACH IN COLUMN CHARTS: The users were interviewed for legend navigation interactive technique in a column chart for all features which are under observation. The user response indicates relatively weak results for effectiveness and usability, while the rest of the features represents encouraging results. About $84 \%$ of the participants agreed that the technique compelled the 
functionality required (Q 4.3), 48\% for effectiveness (Q 8.3), 79\% for efficiency (Q 13.3), $43 \%$ for usability (Q24.3), and 87\% for usefulness (Q 26.3).

Figure 4 illustrates that the legend navigation interactive approach in column charts and shows good results for all visualization features. The bar length in the figure indicates the average response of the users while performing tasks. The standard deviation varies ( 0.49 to 0.63$)$ which shows the stability of the user's responses and reliability of the technique.

5.4.4. LEGEND NAVIGATION APPROACH IN BAR CHARTS: Similarly, to investigate the effectiveness of the legend navigation interactive technique in bar charts, the users were interviewed. The user response indicates relatively weak results for effectiveness and poor results for usability, while the rest of the features represents encouraging results. About $76 \%$ of the participants agreed that the functionality feature (Q 4.4) compelled the user requirement, 39\% for effectiveness (Q 8.4), 77\% for efficiency (Q 13.4), 32\% for usability (Q24.4), and 78\% for usefulness (Q 26.4).

Figure 4 illustrates the effect of visualization features in legend navigation interactive mechanisms in bar charts. After interviewing the participants it has been concluded that features effectiveness and usability represent poor results, it is difficult to efficiently manipulate the interaction in visualization.

5.4.5. DRILL DOWN + LEGEND NAVIGATION APPROACH IN COLUMN CHARTS: The combination of drill-down and legend navigation approach in the column chart presents the most attractive results for visualization features. A good part of participants show their trust, $95 \%$ of the participants agree for the functionality feature $(\mathrm{Q}$ 4.5), 91\% for effectiveness (Q 8.5), 89\% for efficiency (Q 13.5), 86\% for usability (Q24.5), and $81 \%$ for usefulness (Q 26.5).

5.4.6. DRILL DOWN +LEGEND NAVIGATION APPROACH IN BAR CHARTS: The combination of drill down and legend navigation approach in the column chart presents the appealing results for visualization features. $85 \%$ of the participants agreed for the functionality feature (Q 4.6), 89\% for effectiveness (Q 8.6), 84\% for efficiency (Q 13.6), 80\% for usability (Q24.6), and 84\% for usefulness (Q 26.6).

When drill down and legend navigation interactive mechanisms are combined in both column charts and bar charts, it shows promising results for all visualization features as compared to the individual interactive approaches applied.

\subsection{COMPARISON OF MOBILE DEVICES (SMARTPHONES \& TABLETS)}

This section discusses the user's response to mobile devices and compared the results. We compared both smartphones and tablets for the visualization techniques presented using the functional prototype. There are 16 questions addressing mobile devices related features. Q1 is asked about the satisfaction of users serving the task on both the devices, $85 \%$ of the participants are satisfied. Q2 is related to the overall appearance of the visualization techniques, more than $80 \%$ of results achieved, the tablet shows a bit better results. Q3 is about data fetching, manipulation, and navigation time. The participants showed appreciable response and more than $90 \%$ of the selection agreed or strongly agreed. Q4, Q5, Q6, Q7, Q8, and Q9 are regarding each technique, its behavior on mobile devices and the relative results are shown in the table below.

As there is a difference in screen sizes of mobile devices, scalability is one of the main issues. In Q10 the participants asked about the scalability of the visual presentation, shows 93\% healthy user's response. Q11, Q12, Q13 questions address overall attractiveness, focus, attention, and interaction respectively which shows good user's response of approximately $90 \%$ for these features for both smartphones and tablets. Q14, 
Q15, Q16 questions deal with the color combination, title or data or axes labels usability and visibility respectively, tablet (88\%) results are bit better than Smartphone (76\%).

The variability in mobile devices regarding software and hardware support is a challenge; the study considers two types of mobile devices, i.e. smartphone and table. To evaluate the proposed interactive techniques on mobile devices is of utmost importance to examine the utility of the visualization techniques. This section describes how the users rate the interactive techniques on smartphones, tablets.

Figure 5 shows the user's rating regarding all interactive techniques for smartphones. The $\mathrm{LNA}+\mathrm{BC}(\mathrm{M}=3.09$, Me- dian=3, $\mathrm{SD}=0.66)$ technique shows poor results, $\mathrm{DDA}+\mathrm{BC}$ $(\mathrm{M}=3.35$, Median=3, $\mathrm{SD}=0.8)$ shows weak results because mobile device user mostly uses Smartphone as horizontally tilted. If the numbers of bars are large then it looks close, difficult to manipulate and understand due to the height of the Smartphone screen. As the number of bars in LNA is more than the DDA thus it produces poor results. On the other hand, the DDA+LNA+CC present more reliable results on the Smartphone.

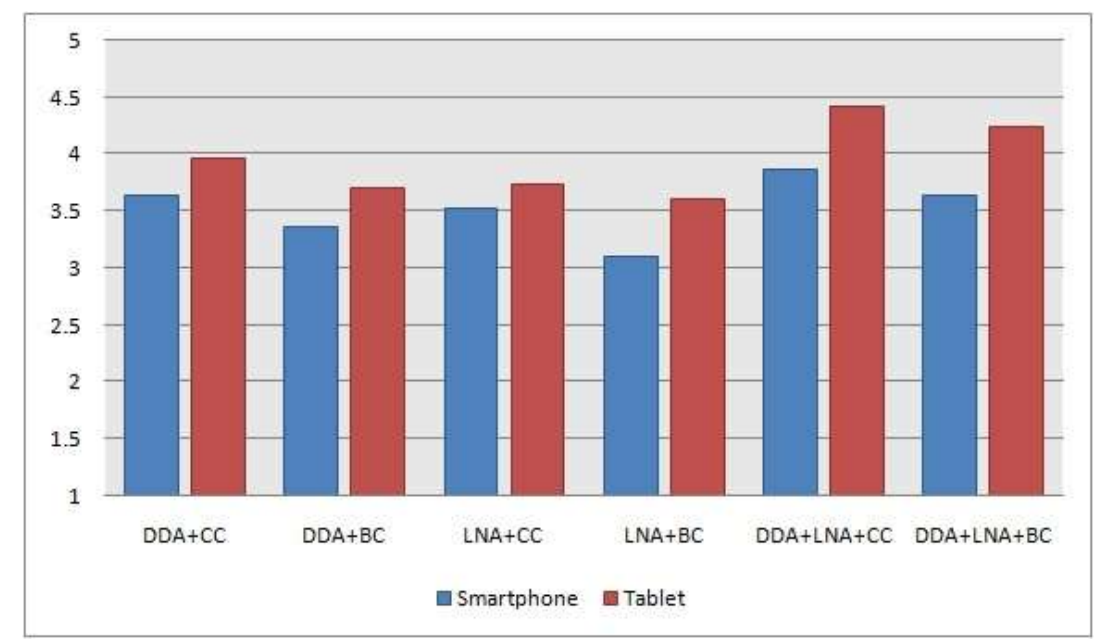

Fig. 5 Comparison: Interactive Visualization Techniques on Mobile Devices

Figure 5 shows the user's rating regarding all interactive techniques which appear to be fair for all the techniques in Tablet. The DDA+LNA+CC and DDA+LNA+BC show the most encouraging results because both techniques provide two ways to explore the information. By DDA one explores the information in more detail (two-level details) and by LNA one explores the comparison with comparative classes, compares different features or characteristics. Other techniques present almost the same results due to a reasonably large screen. The mean values ranges between $\mathrm{M}=3.6$ (Median=4, $\mathrm{SD}=0.62$ ) to $\mathrm{M}=3.97$ (Median=4, $\mathrm{SD}=0.66$ ).

\section{CONCLUSION}

The principal objective of this work is to incorporate the emerging technologies like mobile technology with the advance complicated data analysis techniques and to merge it in such a way that it facilitates a large number of audiences effectively and efficiently with useful information. In the present era and available mobile device resources (computing ability, storage capacity) does not fully support the computationally expensive tasks and massive datasets of data mining, but rather to provide the information or pattern whenever it is required. The best way in the recent situation, client-server architectures allow visualizing or showing the mining results in a significant way for nomadic users using less cognitive resources. 
This study can be summarized as;

The results visualization has many features like the dynamic transformation of layout, layout scalability, the visualization techniques (two techniques) that are used for data characterization and discrimination, multi-brand mobile devices support and interaction mechanisms (three interaction mechanisms) with the visual results, help to explore the information effectively.

- The study introduces six interactive usable visualization techniques for the data mining results, to convey the insight of the data effectively.

- The study involves two visualization techniques, i.e. Column charts (CC) and Bar charts (BC), three interactive mechanisms, i.e. Drill-down approach (DDA), Legend navigation approach (LNA) and drill down combine with legend navigation approach (DDA+LNA). The combination of visualization techniques and interactive mechanisms generates six interactive visualization techniques. In the study, the information visualization technique column charts show reliable results as compared to bar charts, because the bar chart is less common to use as compared to the column chart.

Interactive visualization techniques

- DDA+CC and LNA+CC show reliable and consistent results for all important features regarding information visualization.

- DDA+BC and LNA+BC show fairly good results for functionality, efficiency, and usefulness but less usable and effective to reveal information.

- DDA+LNA+CC and DDA+LNA+BC are highly appreciated for the information dissipation and interaction, which is represented by the most encouraging and promising analysis results.

- Mobile devices (Smartphone and tablet)

- The $\mathrm{LNA}+\mathrm{BC}$ and $\mathrm{DDA}+\mathrm{BC}$ techniques showed poor and weak results respectively for smartphones because users mostly use smartphones as horizontally tilted. If the numbers of bars are large then it looks very close, difficult to manipulate and understand due to the height of the Smartphone screen. As the numbers of bars in LNA are more than the DDA thus it produces poor results, the DDA+LNA+CC presents more reliable results and fair results for other interactive techniques.

- The DDA+LNA+CC and DDA+LNA+BC show the most encouraging results because both the techniques provide two-way information exploration that is; DDA to explore the information in more detail (two-level details) and LNA used to explore the comparison with comparative classes, comparing different features or characteristics. All other techniques present almost the same results due to the reasonably large screen.

These interactive mechanisms can be applied to many other visualization techniques. E.g. to get detail information about an opinion that has been shown by the pie chart in percentage, information like what is the demographics of those give the opinion. On clicking on a portion of the pie chart display inside information using another pie chart or other visualization techniques. In the same way, these interaction techniques or modification can be applied to most of the visualization techniques based on potentially related information available. 


\section{REFERENCES}

[1] Alan Bryman. "The debate about quantitative and qualitative research: a question of method or epistemology?" British Journal of Sociology, 1984, pages 75-92.

[2] Carmela Comito and Domenico Talia. "Energy consumption of data mining algorithms on mobile phones: Evaluation and prediction". Pervasive and Mobile Computing, 2017, Vol. 42, pages 248-264.

[3] D Dacunha-Castelle. "Vedas: A mobile and distributed data stream mining system for real-time vehicle monitoring". 2004.

[4] Ashutosh K Dubey and Shishir K Shandilya. "Exploiting need of data mining services in mobile computing environments". In Computational Intelligence and Communication Networks (CICN), 2010 International Conference on, 2010, pages 409-414. IEEE.

[5] Gregory J Feist and Erika L Rosenberg. "Psychology: perspectives \& connections", 2012 pages 12-08.

[6] Rosemary R Gliem and Joseph A Gliem. "Calculating, interpreting, and reporting Cronbach's alpha reliability coefficient for Likert-type scales". Midwest Research-to-Practice Conference in Adult, Continuing, and Community Education. 2003.

[7] Jiawei Han, Jian Pei, and Micheline Kamber. Data mining: concepts and techniques. 2011, Elsevier.

[8] Nitin Jindal and Bing Liu. "Opinion spam and analysis". In Proceedings of the 2008 International Conference on WebSearch and Data Mining, 2008, pages 219-230, ACM.

[9] Hillol Kargupta, Byung-Hoon Park, Sweta Pittie, Lei Liu, Deepali Kushraj, and Kakali Sarkar "Mobimine: Monitoring the stock market from a PDA". ACM SIGKDD Explorations Newsletter, 2002, 3(2) pages 37-46.

[10] Muzammil Khan. "Interactive Data Mining Results Visualization on Mobile Devices". ISBN 978-3659-46354-9, 2013, LAP USA.

[11] Muzammil Khan, Fida Hussain, and Imran Khan. "Single level drill down interactive visualization technique for descriptive data mining results". International Journal of Grid and Distributed Computing, 2014, 7(4) Pages 33-40.

[12] Muzammil Khan and Sarwar Shah Khan. "Data and information visualization methods, and interactive mechanisms: A survey". International Journal of Computer Applications, 2011, 34(1) Pages 1-14.

[13] Muzammil Khan, Sarwar Shah Khan, and M Daud. "Comparative exploration of data mining results by legend navigation interactive technique". International Journal of Database Theory and Application. 2016, 9(9) Pages 49-58.

[14] Muzammil Khan, Ali Shah, and Israr Ahmad. "Framework for interactive data mining results visualization on mobile devices". International Journal of Database Theory and Application, 2014, 7(4) Pages 23-36.

[15] Ricardo Langner, Tom Horak, and Raimund Dachselt. "Vistiles: Coordinating and combining colocated mobile devices for visual data exploration". IEEE transactions on visualization and computer graphics, 2018, 24(1) Pages 626-636.

[16] Riccardo Mazza and Alessandra Berre. "Focus group methodology for evaluating information visualization techniques and tools". In Information Visualization, 2007. IV'07.11th International Conference, 2007, pages 74-80, IEEE.

[17] Sweta Pittie, Hillol Kargupta, and ByungHoon Park. "Dependency detection in mobimine: a systems perspective. Information Sciences, 2013, 155(3), Pages 227-243.

[18] Calvin Fisher Schmid and Calvin Fisher Schmid. "Statistical graphics; design principles and practices". Technical report, 1983.

[19] Hansi Senaratne, Manuel Mueller, Michael Behrisch, Felipe Lalanne, Javier BustosJim’enez, J"orn Schneidewind, Daniel Keim, and Tobias Schreck. "Urban mobility analysis with mobile network data: A visual analytics approach". IEEE Transactions on Intelligent Transportation Systems, 2018, 19(5), Pages 1537-1546.

[20] Roberto Sousa, Valentina Nisi, and Ian Oakley. "Glaze: A visualization framework for mobile devices". In IFIP Conference on Human-Computer Interaction, 2009, pages 870-873. Springer.

[21] Frederic Stahl, Mohamed Medhat Gaber, Max Bramer, and S Yu Philip. "Pocket data mining: towards collaborative data mining in mobile computing environments". In 22nd IEEE International Conference on Tools with Artificial Intelligence, 2010, volume 2, pages 323-330. IEEE.

[22] Frederic Stahl, Mohamed Medhat Gaber, Max Bramer, and S Yu Philip. "Distributed hoeffding trees for pocket data mining". In High-Performance Computing and Simulation (HPCS), 2011 International Conference on, 2011, pages 686-692. IEEE.

[23] Frederic Stahl, Mohamed Medhat Gaber, Han Liu, Max Bramer, and S Yu Philip. "Distributed classification for pocket data mining". In International Symposium on Methodologies for Intelligent Systems, 2011, pages 336-345, Springer.

[24] Domenico Talia, Paolo Trunfio, et al "Mobile data mining on small devices through web services". Mobile Intelligence, 2010, Pages 69- 64.

[25] Lex Van Velsen, Thea Van Der Geest, Rob Klaassen, and Michael Steehouder. "User-centered evaluation of adaptive and adaptable systems: a literature review". The knowledge engineering review, 2008, 23(03) Pages 261-281. 
[26] Igor Vasconcelos, Rafael Oliveira Vasconcelos, Bruno Olivieri, Marcos Roriz, Markus Endler, and Methanias Cola co Junior. "Smartphone-based outlier detection: a complex event processing approach for driving behavior detection". Journal of Internet Services and Applications, 2017, 8(1) Pages 13.

[27] Frank Wang, Nu Helian, Y Guo, and Hai Jin. "A distributed and mobile data mining system". In Parallel and Distributed Computing, Applications and Technologies. PDCAT'2003. Proceedings of the Fourth International Conference on, 2003, pages 916-918. IEEE. 